\title{
Correction to: From Place-Memories to Active Citizenship: The Potential of Geotagged User-Generated Content for Memory Scholarship
}

\section{Danielle Drozdzewski}

https://doi.org/10.1007/978-981-13-1411-7_8

The chapter, 'From Place-Memories to Active Citizenship: The Potential of Geotagged User-Generated Content for Memory Scholarship' (no. 8) was previously published non-open access, but has now been made available (C) The Author and open access under a CC BY 4.0 license.

The updated online version of this chapter can be found at https://doi.org/10.1007/978-981-13-1411-7_8 